\title{
Dynamics and squeeze film gas damping of a capacitive RF MEMS switch
}

\author{
P.G. Steeneken ${ }^{1}$, Th.G.S.M. Rijks ${ }^{1}$, J.T.M. van Beek ${ }^{1}$, \\ M.J.E. Ulenaers ${ }^{1}$, J. De Coster ${ }^{2}$ and R. Puers ${ }^{2}$ \\ 1 Philips Research Laboratories, Prof. Holstlaan 4, 5656 AA Eindhoven, The \\ Netherlands \\ ${ }^{2}$ K.U.Leuven, Dept. ESAT-MICAS, Kasteelpark Arenberg 10, B-3001 Leuven, \\ Belgium
}

\begin{abstract}
We report on measurements of the time dependent capacitance of a RF MEMS shunt switch. A high time resolution detection setup is used to determine switching time and motion of the device. From the equation of motion the damping force is extracted. The measured damping force is found to be approximately proportional to the speed over the gap distance to the third power $\left(F_{D} \propto v / z^{3}\right)$, in good agreement with squeeze film damping theory. Measurements at low pressure show underdamped harmonic oscillations in the opening motion and contact bounce effects in the closing motion. Effects of dielectric charging on the $\mathrm{C}-\mathrm{V}$ curves are discussed. Experimental results are compared with electromechanical and damping simulations.
\end{abstract}




\section{Introduction}

Research on MEMS (Microelectromechanical Systems) has seen an amazing growth the last 15 years, stimulated both by their interesting physical properties and their attractive application potential. One of those appealing MEMS applications are radio frequency (RF) switches [1]. Advantages of RF MEMS switches over conventional PINdiode switches include lower power consumption, lower insertion loss, higher isolation and good linearity. Despite these advantages several hurdles still have to be overcome to make their application in RF transceiver modules commercially attractive. One of these hurdles is to obtain a sufficiently high switching speed at low actuation voltages.

In many applications, like GSM mobile phones, RF MEMS switches with low actuation voltages and high switching speeds are preferred. However, it is challenging to fulfill both requirements, because for electrostatic switches with low pull-in voltages the switching time tends to increase. This is particularly true for the opening speed of the switch as becomes apparent by rewriting the well known equation for the pull-in voltage[1] to obtain the spring force $F_{\text {open }}$ which is available to open a switch from the closed state:

$$
F_{\text {open }}=k g=\frac{27 V_{P I}^{2} A \varepsilon_{0}}{8 g^{2}}
$$

Here $V_{\mathrm{pi}}$ is the pull-in voltage, $g$ is the electrostatic gap, $A$ the actuation area and $k$ the spring constant of the switch. Equation (1) shows that for constant gap and area, the spring force decreases quadratically with the pull-in voltage, which tends to slow down low pull-in voltage switches.

The main forces reducing the MEMS switching speed are inertial forces which dominate at low pressures, and gas damping forces which often exceed the inertial forces at atmospheric pressure. To obtain sufficiently fast RF MEMS switches it is therefore desirable to understand the effects of gas damping on their motion. This requires accurate models of gas damping to simulate the switching dynamics.

In this paper we present measurements and simulations of the dynamics of a capacitive shunt switch. In particular the damping force during the switching action is examined. The results are compared to squeeze film gas damping models.

\section{The capacitive shunt switch}

A RF MEMS shunt switch was manufactured using the industrialized low-cost Philips' PASSI $^{T M}$ process for passive integration $[2,3]$. The process was extended with a back-end surface micromachining module to realize MEMS devices[4]. A picture of this shunt switch is shown in figure 1. The suspended top electrode is grounded, the bottom electrode carries both the RF signal and the DC actuation voltage. The signal line continues as coplanar waveguide $(\mathrm{CPW})$ on both sides of the switch. The fixed bottom electrode consists of aluminium and the suspended top electrode consists of an aluminum alloy. The spring structure is designed to minimize stresses in the top 


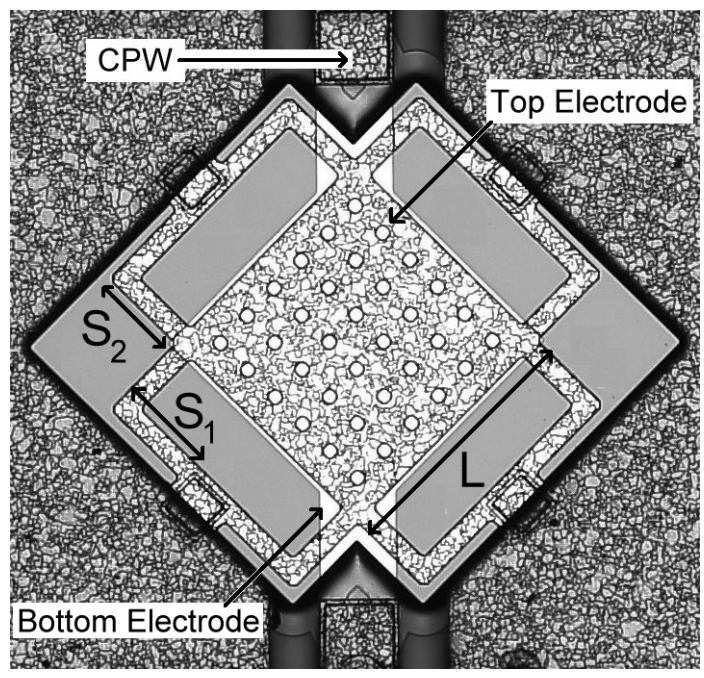

Figure 1. Top view of the capacitive shunt switch.

electrode as a result of thermal fluctuations during manufacturing and operation[5]. Interferometric measurements on the $\mathrm{PASSI}^{T M}$ switch show that the suspended springs and top electrode are very flat, with maximal height deviations of less than $100 \mathrm{~nm}$, an important requirement for accurate modelling of the device. The suspended electrode has a thickness of $4.5 \mu \mathrm{m}$ and the gap between the electrodes is $g=1.5 \mu \mathrm{m}$. As a dielectric the thin native $\mathrm{Al}_{2} \mathrm{O}_{3}$ on the electrodes is used. The capacitance density in the closed state is mainly determined by the surface roughness of the electrodes. It corresponds to the capacitance density of an air gap of $g_{0} \approx 54 \mathrm{~nm}$. Other device dimensions and assumed material properties are summarized in table 1.

\section{High time-resolution capacitance measurement}

Figure 2 shows a schematic of the setup that was used to perform time dependent capacitance measurements. A $1 \mathrm{GHz}$ probing signal is generated by a RF signal generator. Via a bias tee the RF signal is combined with the actuation voltage that is generated by a function generator. RF probes are used to contact the MEMS shunt switch. Via a decoupling capacitor the transmitted RF signal is detected by a calibrated diode power sensor that is connected to an oscilloscope. Due to the high frequency of the probing signal, the short settling time of the diode sensor, and the high sampling rate of the oscilloscope, the time resolution of the setup is better than $10 \mathrm{~ns}$, which allows very accurate measurements of the switching dynamics. We note that in [6] a similar setup was used to measure the switching time.

To extract the switch capacitance from the transmitted power, the $S$-parameters of the connecting circuits (cables, bias tees and probes) on both sides of the switch were measured using a network analyzer. With Mason's rule an equation was derived to relate the transmitted power to the shunt capacitance of the switch and the measured 
Table 1. Properties of the MEMS switch under study.

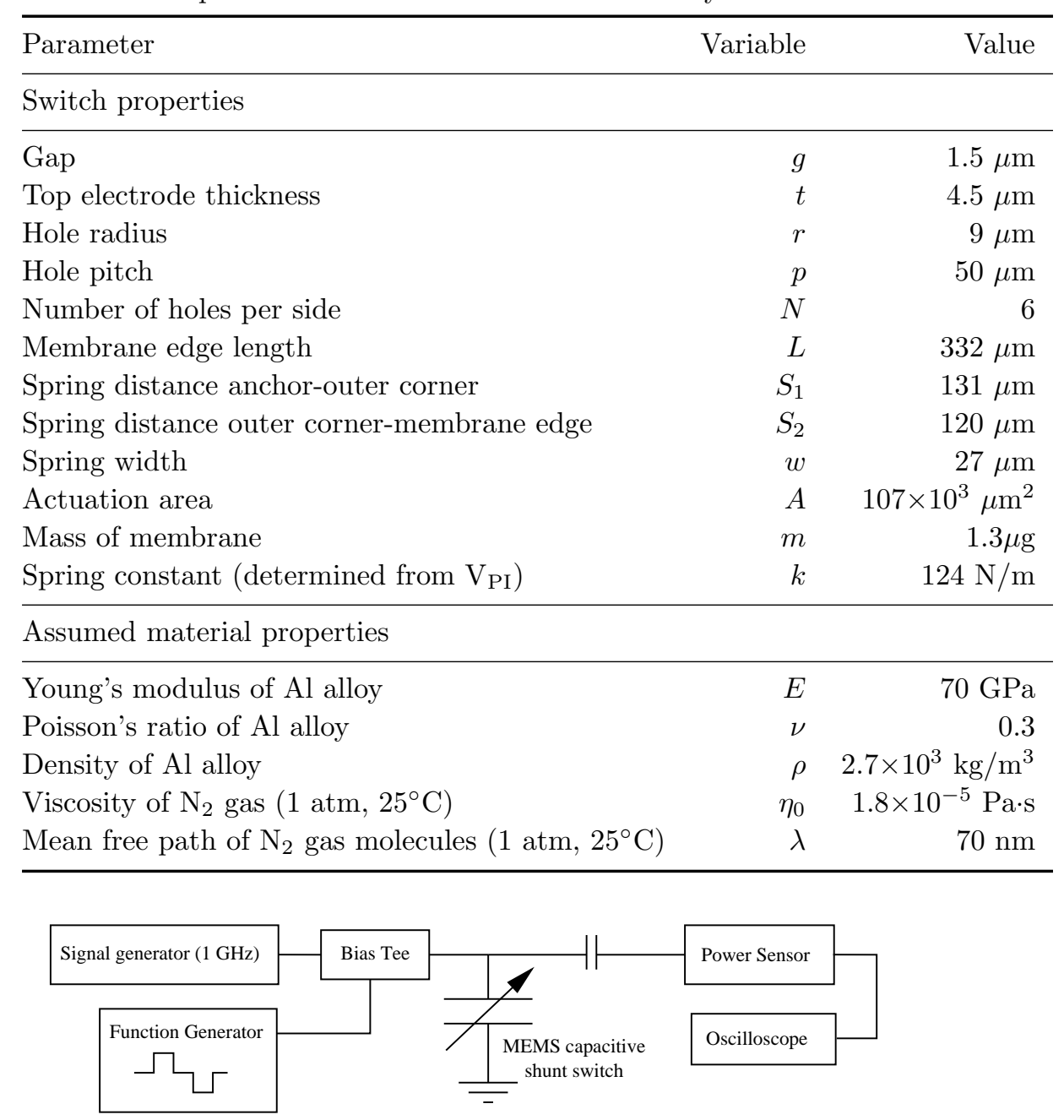

Figure 2. Schematic of the setup that was used to perform the high time-resolution capacitance measurements.

$S$-parameters. By inverting this equation the shunt capacitance as a function of transmitted power was determined. The capacitance between the bottom and top electrode was obtained by subtracting the parallel shunt capacitance of the CPW (202 $\mathrm{fF}$ ), which was determined by measuring the $S$-parameters of an identical device without top electrode.

\section{Quasi-static C-V measurements and charging effects}

$\mathrm{C}-\mathrm{V}$ measurements of the switch were both made by the high time-resolution setup at a probing frequency of $1 \mathrm{GHz}$ and a voltage sweep rate of $450 \mathrm{~V} / \mathrm{s}\left(T_{\text {sweep }}=100 \mathrm{~ms}\right)$ and by using an HP 4275A LCR meter at $1 \mathrm{MHz}$ and a sweep rate of $0.05 \mathrm{~V} / \mathrm{s}\left(T_{\text {sweep }}=15\right.$ minutes). The results are shown in figures $3 \mathrm{a}$ ) and $3 \mathrm{~b}$ ). The $1 \mathrm{MHz}$ measurements were corrected for parasitic substrate capacitances, which become significant at lower 


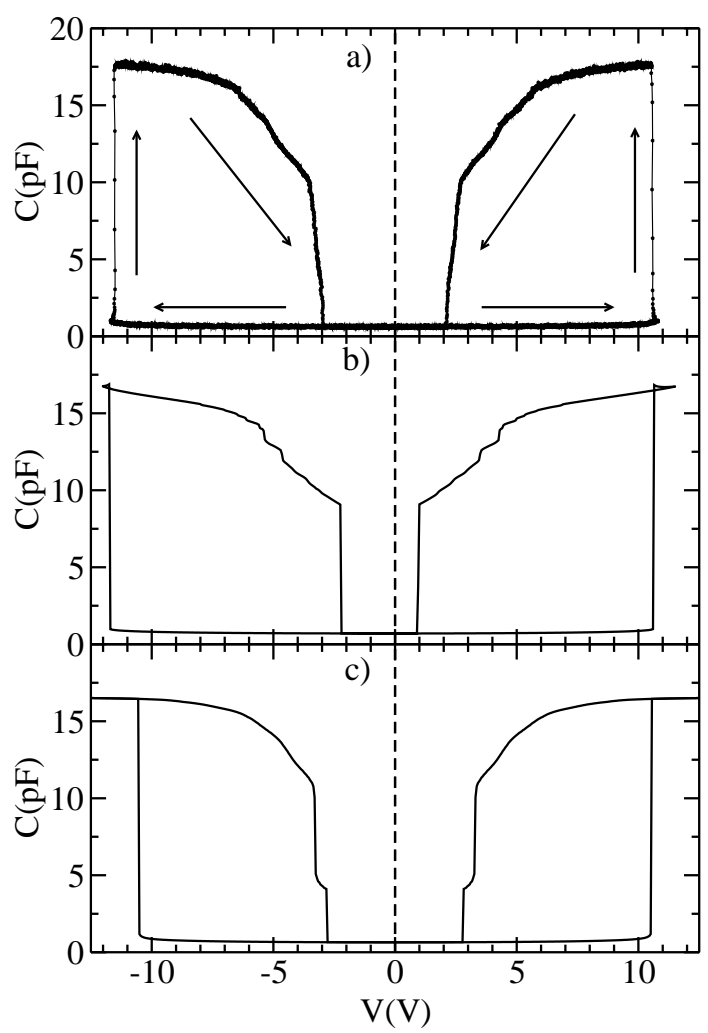

Figure 3. a) C-V measurement at $1 \mathrm{GHz}$ with a fast voltage sweep rate of $450 \mathrm{~V} / \mathrm{s}$. b) $\mathrm{C}-\mathrm{V}$ measurement at $1 \mathrm{MHz}$ with a slow voltage sweep rate of $0.05 \mathrm{~V} / \mathrm{s}$. c) ANSYS simulation of the $\mathrm{C}-\mathrm{V}$ characteristic (static).

frequencies [7].

For comparison a static ANSYS simulation using the geometry and material properties described in table 1 is also shown in figure 3c).

The measurements show a capacitance switching ratio $C_{\text {closed }} / C_{\text {open }}$ of 24 . A shift is observed between the positive and negative pull-in and release voltages. This shift is attributed to charge in the dielectric layer $[8,9]$. To clarify this effect, consider a parallel plate electrostatic actuator containing a surface charge density $\sigma_{s}$ on top of its dielectric as shown in figure 4.

Applying Gauss' law $\left(\vec{\nabla} \cdot \vec{D}=\rho_{f}\right)$ and $\vec{\nabla} V=-\vec{E}$ it can be shown that the surface charge density on the top metal $\sigma_{u}$ is given by:

$$
\sigma_{u}=\frac{V-\sigma_{s} z_{d} / \varepsilon_{d}}{z / \varepsilon_{0}+z_{d} / \varepsilon_{d}}
$$

As the electrostatic force per unit area on a conductor with surface charge density $\sigma_{u}$ and area $A$ is [10],

$$
F_{e l}=\frac{-A \sigma_{u}^{2}}{2 \varepsilon_{0}}
$$

it follows that the presence of a surface charge results in a shift in the $\mathrm{C}-\mathrm{V}$ curve which can be accounted for by an effective actuation voltage $V_{\text {eff }}=V-\Delta$ with $\Delta=\sigma_{s} z_{d} / \varepsilon_{d}$. 


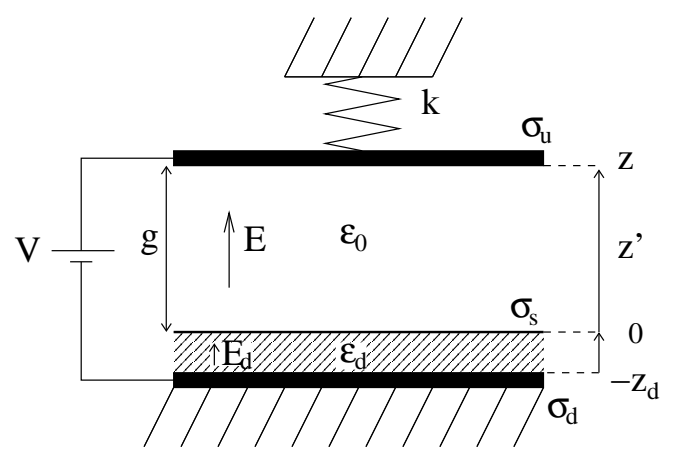

Figure 4. Simplified model of an electrostatic actuator. A dielectric with thickness $z_{d}$, dielectric constant $\varepsilon_{d}=\varepsilon_{0} \varepsilon_{d, r}$ and surface charge density $\sigma_{s}$ covers the bottom electrode.

Table 2. Pull-in, release and shift voltages of the switch as determined from the C-V characteristics in figure 3 .

\begin{tabular}{lrrrr}
\hline & $V_{P I}^{\mathrm{eff}}(\mathrm{V})$ & $\Delta_{P I}(\mathrm{~V})$ & $V_{\text {rel }}^{\mathrm{eff}}(\mathrm{V})$ & $\Delta_{\text {rel }}(\mathrm{V})$ \\
\hline $1 \mathrm{GHz}, 450 \mathrm{~V} / \mathrm{s}$ & 11.08 & -0.48 & 2.53 & -0.43 \\
$1 \mathrm{MHz}, 0.05 \mathrm{~V} / \mathrm{s}$ & 11.20 & -0.55 & 1.55 & -0.65 \\
ANSYS & 10.56 & & 2.77 & \\
\hline
\end{tabular}

Therefore the pull-in and release voltages of the switch are also shifted and become:

$$
V_{P I}^{ \pm}=\Delta \pm \sqrt{\frac{8 k}{27 A \varepsilon_{0}}\left(g+\frac{\varepsilon_{0}}{\varepsilon_{d}} z_{d}\right)^{3}} \quad \text { and } \quad V_{r e l}^{ \pm}=\Delta \pm \frac{z_{d}}{\varepsilon_{d}} \sqrt{\frac{2 g k \varepsilon_{0}}{A}}
$$

It is straightforward to extend this to an arbitrary vertical charge density distribution $\rho_{d}\left(z^{\prime}\right)$ inside the dielectric, for which the voltage shift $\Delta$ becomes:

$$
\Delta=\frac{1}{\varepsilon_{d}} \int_{-z_{d}}^{0}\left(z^{\prime}+z_{d}\right) \rho_{d}\left(z^{\prime}\right) \mathrm{d} z^{\prime} \equiv \frac{\sigma_{s}^{\text {eff }} z_{d}}{\varepsilon_{d}}
$$

The mathematical identity $\int_{0}^{x} \mathrm{~d} x_{2} \int_{0}^{x_{2}} f\left(x_{1}\right) \mathrm{d} x_{1}=\int_{0}^{x}(x-t) f(t) \mathrm{d} t$ is used to obtain this expression. The effective voltage shift for an arbitrary vertical charge distribution is therefore a weighted average over the charge, where charge nearest to the surface of the dielectric contributes most to the shift.

Positive and negative pull-in and release voltages in figure 3 were used to determine the effective pull-in and release voltages $V_{P I / \text { rel }}^{\text {eff }}=\left(V_{P I / r e l}^{+}-V_{P I / r e l}^{-} / 2\right)$ and the voltage shifts $\Delta_{P I / r e l}=\left(V_{P I / r e l}^{+}+V_{P I / r e l}^{-} / 2\right)$. The resulting values are shown in table 2 .

The effective pull-in voltages of the fast voltage sweep measurement and slow sweep measurement are only $0.1 \mathrm{~V}$ different. Also the voltage shift $\Delta$ for pull-in and release are quite similar for both sweep rates. However the effective release voltage of the slow rate sweep is nearly $1 \mathrm{~V}$ lower than that of the fast sweep measurement. A possible cause for this reduction of $\left|V_{\text {rel }}^{\text {eff }}\right|$ might be a voltage dependent charge injection $\pm \sigma_{i n j}^{\text {eff }}$ into the dielectric. Charge injection will most likely occur when the switch is closed and will increase with time. When the switch is open, the electric field in the 
dielectric is much lower, allowing the injected charge to (partially) leak away. As a result of charge injection $\pm \sigma_{i n j}^{\text {eff }}$ in the closed state we find a shift in release volage $\Delta_{\text {rel }}^{ \pm}=\left(\sigma_{s}^{\text {eff }} \pm \sigma_{i n j}^{\text {eff }}\right) z_{d} / \varepsilon_{d}$ resulting in a shift in $V_{r e l}^{\text {eff }}$ of $\Delta_{i n j}=\sigma_{i n j}^{\text {eff }} z_{d} / \varepsilon_{d}$. The observed reduction in release voltage of $-1 \mathrm{~V}$ at slow sweep rate therefore corresponds to an additional injected charge density of $\sigma_{i n j}^{\text {eff }}=-16 \mathrm{mC} / \mathrm{m}^{2}$, the fixed charge density is approximately $\sigma_{s}^{\text {eff }} \approx-8 \mathrm{mC} / \mathrm{m}^{2}\left(\sim 1000\right.$ electrons $\left./ \mu \mathrm{m}^{2}\right)$. Recently lateral charge variations in $\sigma_{s}^{\text {eff }}(x, y)$ were proposed as an alternative mechanism to reduce $\left|V_{r e l}^{\text {eff }}\right|$ [11]. In the limit that the lateral correlation length $\xi$ (i.e. the 'wavelength' of the variations) of this lateral distribution is much larger than the plate distance $z$, it was shown that for a normal distributed surface charge density with average $\overline{\sigma_{s}^{\text {erf }}}$ and standard deviation $s\left(\sigma_{s}^{\text {eff }}\right)$, the electrostatic force is given by:

$$
F_{e l}=\frac{-A\left[\left(V-\overline{\sigma_{s}^{\text {eff }}} z_{d} / \varepsilon_{d}\right)^{2}+\left(s\left(\sigma_{s}^{\mathrm{eff}}\right) z_{d} / \varepsilon_{d}\right)^{2}\right]}{2 \varepsilon_{0}\left(z / \varepsilon_{0}+z_{d} / \varepsilon_{d}\right)^{2}}
$$

This equation shows that charge variations increase the electrostatic force and thus result in 'narrowing' of the $\mathrm{C}-\mathrm{V}$ characteristic and a reduction of $\left|V_{P I / \text { rel }}\right|$, whereas the average charge density leads to a shift in $\mathrm{C}-\mathrm{V}$ curve. If $\xi<<z$ the electric field due to the lateral charge variations will average out at the top electrode and the lateral variations will not affect the $\mathrm{C}-\mathrm{V}$ characteristic.

From the measurements we conclude that $\mathrm{C}-\mathrm{V}$ measurements with a fast voltage sweep rate seem to be less sensitive to charging effects than those with slow sweep rate which is favorable for modelling purposes. On the other hand, to stay in the quasistatic regime, the sweep time should not be decreased too much and should be kept much larger than the switching time. Otherwise transient switching effects will affect the shape of the curve.

The ANSYS finite element simulation in figure 3c) captures the shape of the C$\mathrm{V}$ curve quite well. Also the calculated pull-in and release voltage are similar to the measured values. The small differences between measurement and simulation are probably due to differences between the assumed and actual material properties, prestress effects and charging, which were not included in the simulation.

\section{Dynamic capacitance measurements}

The switching characteristics of the switch are studied using the setup described in section 3. With the function generator a block function voltage just above the pull-in voltage $\left(V_{\text {eff }}=V_{P I}^{\text {eff }}+0.1 \mathrm{~V}=11.2 \mathrm{~V}\right)$ is applied at $t=0$ and the subsequent capacitance change of the switch is monitored to study the closing dynamics of the switch. Similarly the opening dynamics are studied when the voltage is set to $V_{\text {eff }}=0$. In figure 5 the time dependent capacitance of the switch during the opening and closing motion is shown. It is found that the switch opens in $550 \mu \mathrm{s}$ and closes in $950 \mu \mathrm{s}$. Note that a small capacitance 'spike' occurs at $t=0$ which is an artifact as a result of the (de)charging of the decoupling capacitor. 


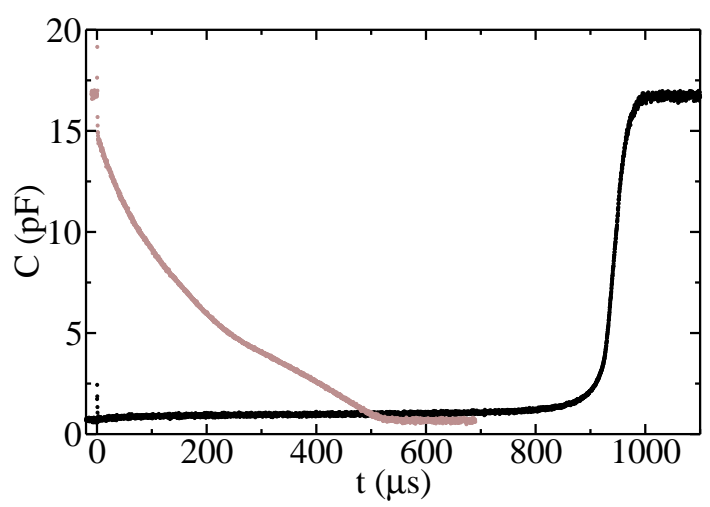

Figure 5. Time dependent capacitance during the opening (grey) and closing (black) of the switch at atmospheric pressure.

To accurately extract the position of the switch from the capacitance, fringing fields need to be taken into consideration. Because the bottom electrode extends much $(\gg g)$ beyond the edge of the top electrode, we can approximate the fringing fields of the top electrode by a microstripline with width $W_{s}$ and gap $z$. Using conformal mapping techniques it can be shown [12] that the factor $\gamma(z)$ by which the capacitance increases with respect to the parallel plate capacitance $\left(C_{\mathrm{pp}}=A \varepsilon_{0} / z\right)$ as a result of fringing fields is given by $\ddagger$ :

$$
\gamma(z)=\frac{C_{\text {fringe }}}{C_{\mathrm{pp}}}=1+\frac{2 z}{\pi W_{s}}\left[1+\ln \left(\frac{\pi W_{s}}{2 z}\right)\right]
$$

As fringing fields only arise at the edges, the fraction $\gamma(z)$ depends mainly on the circumference and area of the top electrode. By equating the circumferences of the microstripline and the actual actuation electrode, $2 L_{s}^{\text {eff }}=4 L+2 N^{2} \pi r$, we obtain the effective stripline length $L_{s}$ and by equating the areas we obtain the effective width $W_{s}^{\text {eff }}=A / L_{s}^{\text {eff }}$. For the switch under study $W_{s}^{\text {eff }}=63.6 \mu \mathrm{m}$. Using equation (7) we find that the fringing field factor $\gamma(z)$ reduces from 1.08 in the open state to 1.005 in the closed state. If the top metal of the switch stays approximately flat, the position of the switch $z$ can be obtained from the measured capacitance $C_{\text {meas }}$ by numerically solving the equation:

$$
z=\frac{\gamma(z) A \varepsilon_{0}}{C_{\text {meas }}}
$$

Using equation (8) the motion of the switch at atmospheric pressure is extracted from the data in figure 5 and is plotted in figure 6 . During the opening motion, the speed of the switch increases monotonically until it reaches the open position. The absence of overshoot indicates that the switch is overdamped at atmospheric pressure. During the closing motion, the speed of the switch first decreases until it passes the pull-in point $\left(z_{P I} \approx 2 g / 3=1 \mu \mathrm{m}\right)$ after which it accelerates and reaches the bottom electrode. Also $\ddagger$ To simplify expressions, from here on $z$ is assumed to include the equivalent air gap in the closed state (i.e. $z+\frac{\varepsilon_{0}}{\varepsilon_{d}} z_{d}$ will be implicitly replaced by $z$ ). 


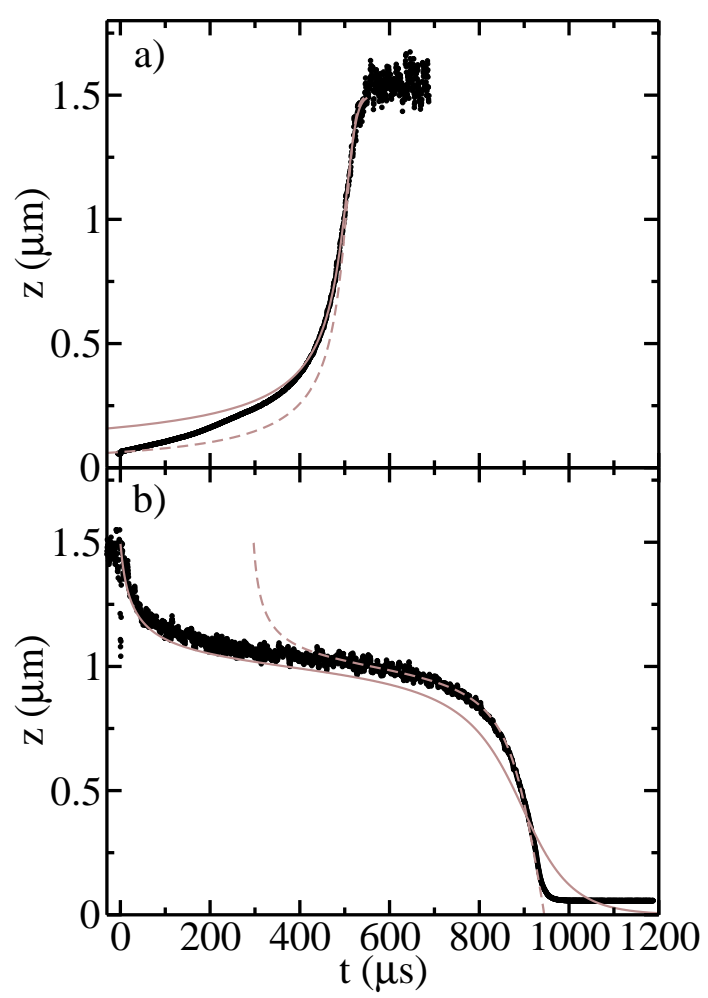

Figure 6. Motion of the top metal during a) opening and b) closing of the switch at atmospheric pressure (black circles). Also shown are analytic solutions equations (18) and (19)) including slip-flow (grey dashed lines) and without slip-flow (grey solid lines).

shown in figure 5 are the theoretical switching curves which will be discussed in the next section.

\section{Squeeze film damping}

\subsection{Damping force}

Squeeze film damping effects in MEMS have been thoroughly studied for small amplitude oscillations around an equilibrium position both theoretically and experimentally $[13,14,15,16,17,18,19,20,21]$. In recent work, measurements and models of the switching time and motion of MEMS switches under the influence of squeeze film damping have also been investigated [22, 23, 24, 25, 26, 27, 6, 28]. However to our knowledge no measurements of the gas damping force $F_{D}$ on MEMS switches as a function of position have been performed.

The high time-resolution measurements allow us to determine this position and speed dependent gas damping force $F_{D}$ during switch motion. By differentiation of the measured motion $z(t)$, the velocity $v(t)$ and acceleration $a(t)$ can be calculated. Neglecting deformations of the center plate during switching, the spring force $F_{s}$, inertial 
force $F_{i}$ and electrostatic force $F_{e l}$ are well approximated by:

$$
\begin{aligned}
& F_{s}=k(g-z) \\
& \text { and } F_{e l}=\frac{\mathrm{d}}{\mathrm{d} z}\left(\frac{\gamma(z) A \varepsilon_{0} V_{\mathrm{eff}}^{2}}{2 z}\right)=-A \varepsilon_{0} V_{\text {eff }}^{2}\left(\frac{1}{2 z^{2}}+\frac{1}{\pi W_{s} z}\right)
\end{aligned}
$$

From the condition that the sum of the forces on the top metal is zero, it is thus possible to extract the damping force on the switch from the measured motion using:

$$
F_{D}=A \varepsilon_{0} V_{\mathrm{eff}}^{2}\left(\frac{1}{2 z^{2}}+\frac{1}{\pi W_{s} z}\right)+m a-k(g-z)
$$

In section 6.5 equation (10) will be used to determine the experimental damping force and compare it to squeeze film models.

\subsection{Simplified Reynolds equation}

In general gas damping of MEMS switches is described by the Navier-Stokes equations, which simplify to the Reynolds equation under the conditions that the gap is small compared to the lateral dimensions $(g \ll(p-2 r))$, inertial effects of the gas can be neglected, the gas is isothermal and acts like an ideal gas. If in addition the membrane stays approximately flat and the pressure variations in the film are considerably smaller than the ambient pressure, the pressure distribution $P_{t}(x, y)$ in the squeezed gas film is described by the simplified Reynolds equation (11), where $P(x, y)=P_{t}(x, y)-P_{0}$ is the difference between the film pressure and the ambient pressure $P_{0}$.

$$
\nabla^{2} P(x, y)=\frac{\partial^{2} P}{\partial x^{2}}+\frac{\partial^{2} P}{\partial y^{2}}=-\frac{12 \eta v}{z^{3}}
$$

where $\eta$ is the effective viscosity. Because the maximum pressure exerted by the spring force of the switch $F_{s, \max } / A=\mathrm{kg} / A=1.7 \mathrm{kPa}$ is indeed always less than $2 \%$ of the ambient pressure, the condition that pressure variations under the membrane are small is satisfied for the opening of the switch. During the closing motion the electrostatic pressure $F_{e l} / A$ stays below $10 \%$ of the ambient pressure for $z>240 \mathrm{~nm}$. For smaller gaps deviations from equation (11) might be expected. Because the diameter of the holes in the switch is much larger than the gap below the switch the contribution of the pressure drop between the top and bottom of the holes to the damping force can be

safely neglected. Thus the boundary condition at the edges of the switch and the holes can be taken as $P_{\text {edge }}=0$.

As the right side of equation (11) does not depend on the lateral position, $P(x, y)=-\left(12 \eta v / z^{3}\right) \mathcal{P}(x, y)$, where $\mathcal{P}(x, y)$ only depends on the lateral geometry of the switch. The total squeeze film damping force on the switch is therefore given by:

$$
F_{D}=\int_{A} P(x, y) \mathrm{d} x \mathrm{~d} y=-12 \eta \frac{v}{z^{3}} \int_{A} \mathcal{P}(x, y) \mathrm{d} x \mathrm{~d} y \equiv-b \frac{v}{z^{3}}
$$

Here $b$ is defined as the squeeze film damping constant. For our switch we have calculated $b_{\mathrm{FEM}}$ in ANSYS, by solving equation (11) using an internal heat generation analogy[16]. The resulting pressure distribution is shown in figure 7 . Integration over the pressure yielded $b_{\mathrm{FEM}} / \eta=2.24 \times 10^{-16} \mathrm{~m}^{4}$. 


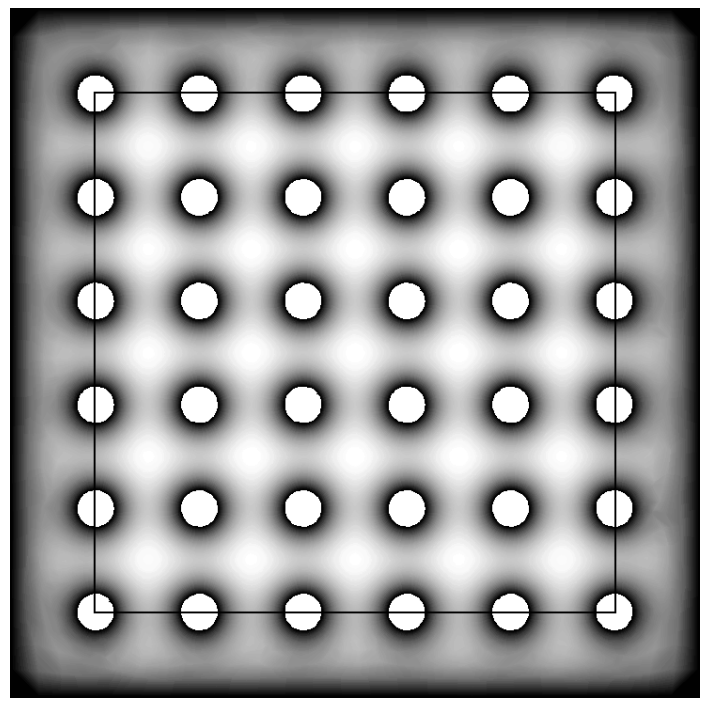

Figure 7. Calculated pressure distribution $P(x, y)$ under the switch membrane. White is maximum pressure, black is zero pressure.

\subsection{Analytic expression for damping coefficient}

For quick simulation it is convenient to have an analytic expression to estimate $b$. Using equation (11), Škvor has calculated the gas damping of a circular disk with a concentric hole, where gas can only escape through the hole[29, 1]. For a plate with $N_{s}^{2}$ holes this yielded:

$$
b_{\text {skvor }}\left(A_{t}, A_{h}, N_{s}\right)=\frac{12 \eta A_{t}^{2}}{N_{s}^{2} \pi}\left(\frac{A_{h}}{2 A_{t}}-\frac{A_{h}^{2}}{8 A_{t}^{2}}-\frac{1}{4} \ln \left(\frac{A_{h}}{A_{t}}\right)-\frac{3}{8}\right)
$$

Here $A_{t}=L^{2}$ is area of the plate including the holes, and $A_{h}$ is the area of the holes. Applying this equation to our switch we find $b_{\text {skvor }} / \eta=3.7 \times 10^{-16} \mathrm{~m}^{4}$. Škvor's equation largely overestimates the damping constant because it doesn't take into account gas flow from the outer edges of the membrane. To include edge effects, we propose to use Škvor's equation only for the center of the membrane (indicated by the black square in figure 7). To calculate the damping of the edge of the membrane (outside the black square) we approximate it by a rectangular plate of effective dimensions $W_{e}$ by $L_{e}$, with equal area $A_{e}$ and circumference $C_{e}$ as the edge region. Figure 2 in Starr's paper[16] shows that the damping under a rectangular plate is approximately given by:

$$
b_{\text {rectangle }}\left(L_{e}, W_{e}\right)=\eta\left(L_{e}-0.6 W_{e}\right) W_{e}^{3}
$$

Therefore we find for squeeze film damping of a perforated plate including edge effects:

$$
b_{\text {perf. }}=b_{\text {skvor }}\left(A_{t}, A_{h}, N_{s}\right)+b_{\text {rectangle }}\left(L_{e}, W_{e}\right)
$$

For the switch under study $A_{t}=(N-1)^{2} p^{2}, A_{h}=(N-1)^{2} \pi r^{2}, N_{s}=N-1$, $A_{e}=L^{2}-A_{t}-(2 N-1) \pi r^{2}, C_{e}=4(L+(N-1 / 2) \pi r), L_{e}=\left(C_{e}+\sqrt{C_{e}^{2}-16 A_{e}}\right) / 4$ and $W_{e}=A_{e} / L_{e}$. Thus we find $b_{\text {perf }} / \eta=2.49 \times 10^{-16} \mathrm{~m}^{4}$ which is only $11 \%$ higher than the finite element solution $b_{\mathrm{FEM}}$. 


\subsection{Slip-flow effects}

If the gap dimensions are of the order of the mean free path of the gas molecules $\lambda$, the no-slip boundary condition at the interface between solid and gas becomes invalid and slip-flow effects need to be considered. The importance of these effects is indicated by the Knudsen number $K_{n}=\lambda / z$, where the mean free path of gas molecules $\lambda \approx 70 \mathrm{~nm}$ under atmospheric pressure at room temperature. Veijola[18] has proposed a modification of the effective viscosity to account for slip-flow:

$$
\frac{\eta_{\mathrm{eff}}}{\eta_{0}}=\frac{1}{1+9.638 K_{n}^{1.159}} \approx \frac{1}{1+\kappa g / z}
$$

In the next subsection this equation will be compared to the measured squeeze film damping force. The approximation on the right side of the equation with $\kappa=9 \lambda / g$ will be used to obtain an analytic expression for the motion in section 7 . In the operating regime of the switch, the maximum error made by this approximation is $12 \%$.

\subsection{Measurement of squeeze film damping}

To compare the theoretically expected damping force of equation (12) with the experimentally determined damping force using equation (10), we have plotted $\left(\frac{-F_{D}(z)}{v(z)}\right)^{-1 / 3}$ in figure 8. Equation (11) shows that in the absence of slip flow effects a linear relation $\left(\frac{-F_{D}(z)}{v(z)}\right)^{-1 / 3}=b^{-1 / 3} z$ is expected, as indicated by the solid line. The presence of slip-flow effects results in a reduction of the damping force as is shown by the dashed line which uses equation (16) for the effective viscosity.

The overall agreement between measurement and theory as shown in figure 8 is good, which suggests that the damping force as described in equation (12) captures most of the squeeze film damping physics at atmospheric pressure. Because the speed of the switch during closing is very low for $0.8 \mu \mathrm{m}<z<1.1 \mu \mathrm{m}$, measurement noise is amplified in this part of plot 8b). At small values of $z$ differences between measurement and theory might be introduced by bending of the top electrode. At small gaps the membrane can still be partly touching the bottom electrode, leading to an increased spring force $F_{s}$, which is not taken into account when extracting the damping force using equation (10). This possibly explains the kink at $z=230 \mathrm{~nm}$ in the opening curve of figure 8a). Because the speed $v$ has opposite sign during opening and closing, the increased spring constant results in opposite deviations of the curves in figure 8 at small $z$.

An interesting observation is that damping during the opening motion seems to be better approximated by the no slip-flow viscosity curve (solid line), whereas the damping during closing is better approximated by slip-flow damping viscosity (dashed line). However these differences might still be within the measurement uncertainties. 


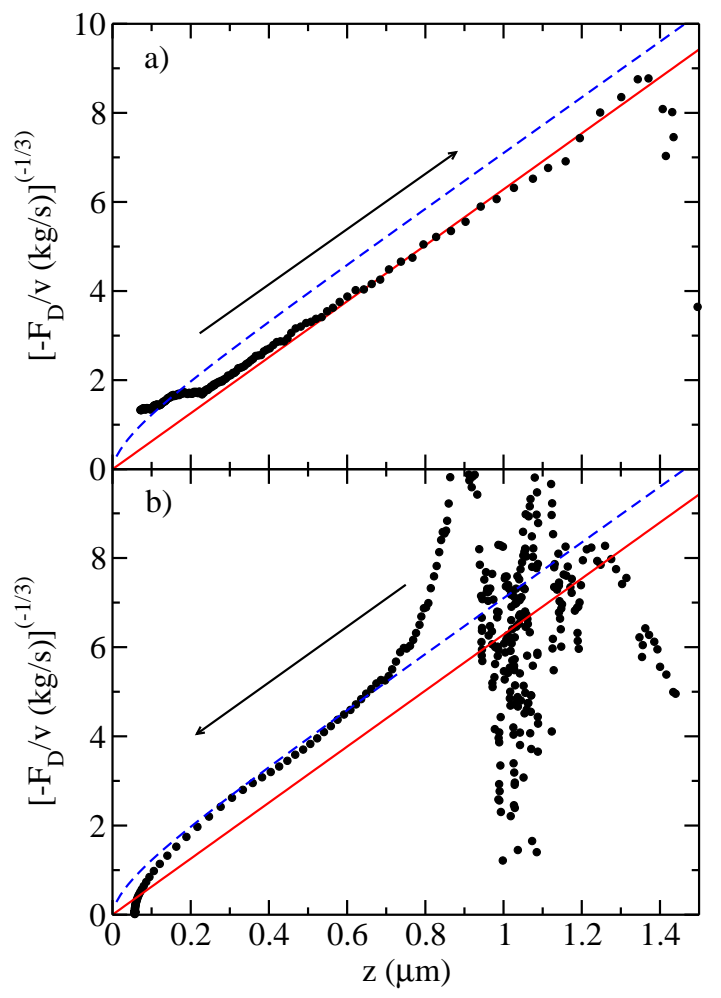

Figure 8. Measured damping curve $\left(\frac{-F_{D}(z)}{v(z)}\right)^{-1 / 3}$ (black circles) during a) opening and b) closing of the switch at atmospheric pressure. Also shown are the damping curves based on the calculated damping coefficient $b_{\mathrm{FEM}}$ in the case of no slip flow (solid) and based on the slip flow equation (16) (dashed).

\section{Equation of motion}

The agreement between the measured and calculated damping force shows that the motion of the electrostatic MEMS switch is well approximated by the following equation of motion:

$$
m a+\frac{b}{(1+\kappa g / z)} \frac{v}{z^{3}}+k(z-g)+A \varepsilon_{0} V_{\mathrm{eff}}^{2}\left(\frac{1}{2 z^{2}}+\frac{1}{\pi W_{s} z}\right)=0
$$

This equation can facilitate rapid modelling of the switch motion and switching time.

\subsection{Analytic solution}

In fact, if inertial effects and fringe fields can be neglected, analytic expressions for the motion including slip-flow can be obtained from equation (17) by integration. For the opening motion $\left(V_{\text {eff }}=0\right)$, from the closed $\left(\tilde{z_{0}}\right)$ to the open $\left(\tilde{z_{1}}\right)$ position we find:

$$
\left.t_{\text {open }}(\tilde{z})\right|_{\tilde{z}_{0}} ^{\tilde{1}_{1}}=\left.\frac{-8 b}{27 A \varepsilon_{0} V_{\mathrm{PI}}^{2}(1+\kappa)}\left[\frac{1+\kappa}{\kappa \tilde{z}}+\ln \left(\frac{1}{\tilde{z}}-1\right)-\frac{1}{\kappa^{2}} \ln \left(1+\frac{\kappa}{\tilde{z}}\right)\right]\right|_{\tilde{z}_{0}} ^{\tilde{z}_{1}}(18
$$


Where the normalized position $\tilde{z}=z / g$ and the slip-flow constant $\kappa=9 \lambda / g$, from equation (16). For the closing motion $\left(V_{\text {eff }}>V_{\mathrm{PI}}\right)$ we find:

$$
\begin{aligned}
& \left.t_{\text {close }}(\tilde{z})\right|_{\tilde{z}_{1}} ^{\tilde{z_{0}}}=\frac{-8 b}{A \varepsilon_{0} V_{\mathrm{PI}}^{2} f_{1}(-\kappa) f_{2}(-\kappa) f_{3}(2 \delta)} \times \\
& {\left[f_{3}(2 \delta) \ln (\tilde{z}+\kappa)-\frac{f_{1}(-\kappa)}{3} \ln \left(f_{2}(\tilde{z})\right)+\right.} \\
& \frac{f_{1}(-\kappa)-3 f_{3}(2 \delta)}{6} \ln \left(f_{1}(\tilde{z})\right)- \\
& \left.\frac{f_{2}(-\kappa)\left(f_{2}(-\delta \kappa)-3 \delta\right)}{\sqrt{3 f_{3}(\delta)}} \arctan \frac{f_{2}(\tilde{z})-3 \delta}{\sqrt{3 f_{3}(\delta)}}\right]\left.\right|_{\tilde{z_{1}}} ^{\tilde{z_{0}}} \\
& \text { with: } \delta=\cosh \left[\frac{1}{3} \operatorname{arccosh}\left(\frac{2 V_{\mathrm{eff}}^{2}}{V_{\mathrm{PI}}^{2}}-1\right)\right] \\
& f_{1}(x) \quad=f_{2}(x)\left(f_{2}(x)-6 \delta\right)+3 f_{3}(2 \delta) \\
& f_{2}(x) \quad=3 x+2 \delta-1 \\
& f_{3}(x)=x^{2}-1
\end{aligned}
$$

\subsection{Comparison with experiment}

As the inertial forces are indeed much smaller than the damping forces at atmospheric pressure, the equations (18) and (19) describe the motion of the switch quite well as long as the damping follows the solid line in figure 8. For comparison the equations have been plotted in figure 6 using $b_{\mathrm{FEM}}$ and $V_{\text {eff }}=11.2 \mathrm{~V}$. Both the analytic curves with (dashed line) and without slip-flow effects (solid line) have been plotted including a shift along the time axis to facilitate comparison. Figure 6a) shows that the calculated motion without slip-flow is in excellent agreement with the measured motion for $z>400$ $\mathrm{nm}$. For smaller $z$ the damping is overestimated, in correspondence with the findings in figure 8a). The calculated opening curve including slip-flow deviates slightly from the measured curve. This might partially be a result of the neglect of membrane bending. From figure 6b) we see that the analytic expression without slip-flow describes the initial motion quite well but overestimates the damping for $z<1 \mu \mathrm{m}$. The curve including slipflow follows the measured curve very closely at small gaps, but closes slightly too fast in the initial stage. Small uncertainties in $\mathrm{V}_{\text {eff }}( \pm 0.05 \mathrm{~V})$ and the high sensitivity of the switching time to $V_{\text {eff }}$ near the pull-in voltage might be responsible for the differences.

\subsection{Conclusions}

Equations (18) and (19) both have the same prefactor $t_{n}=8 b /\left(27 A \varepsilon_{0} V_{\mathrm{PI}}^{2}\right)=b /\left(k g^{3}\right)$, which is $9.6 \mu \mathrm{s}$ for the switch under study. This shows that the switching time can be reduced by increasing $V_{\mathrm{PI}}$. Also decreasing the squeeze film damping constant $b$ by increasing the (number of) holes in the membrane or by reducing the pressure increases the speed. Note that increasing the membrane area does not decrease the switching 
time as for a constant hole density $b \propto A$. In the limit of large gas damping, the normalized switching time $\tilde{t}=t / t_{n}$ depends on just three parameters: $\kappa, V_{\text {eff }} / V_{\text {PI }}$ and the normalized air gap in the closed position $\tilde{z_{0}}$. This implies that the $\tilde{z}(\tilde{t})$ curve will be identical as long as these three parameters are kept constant.

Further inspection of equation (18) learns that in the limit of small roughness $\tilde{z}_{0}$, the logarithmic terms can be neglected and we find for the normalized opening time:

$$
\lim _{\tilde{z}_{0} \rightarrow 0} \tilde{t}_{\text {open }}=\frac{1}{\tilde{z}_{0}}
$$

This shows that without roughness it would take infinitely long for the switch to open. Moreover it relates the maximum capacitance tuning ratio to the opening time. If the capacitance density in the closed state is mainly determined by the thickness of the air gap $z_{0}$, the opening time for large capacitance ratios will be given by $t_{\text {open }} \approx t_{n} C_{\text {closed }} / C_{\text {open }}$.

\section{Motion at low pressure}

Capacitance measurements of the opening and closing motion were also performed at a pressure of 1.4 mbar. Using the method outlined in section 5, the motion was extracted as is shown in figure 9. The opening motion in figure 9a) shows that the switch exhibits a strongly underdamped harmonic oscillation, as expected for a mass-spring system with low damping constant. The average oscillation period $\mathrm{T}_{\text {osc }}$ during the first 9 oscillations is $20.7 \mu \mathrm{s}$. Note that at this low pressure an opening time (i.e. the time in which the switch reaches $z=g$ ) of only $\mathrm{T}_{\text {open }}=4.7 \mu \mathrm{s} \approx \mathrm{T}_{\text {osc }} / 4$ has been measured.

The closing time is also strongly reduced at low pressures. Figure $9 \mathrm{~b}$ ) shows that the switch closes in $17.5 \mu \mathrm{s}$ for $V_{\text {eff }}=11.2 \mathrm{~V}$. Zooming in at the motion after the switch has collapsed, shows a periodic bouncing effect (inset of figure 9) which is rather irregular and can be observed for more than $40 \mu$ s after the switch has made contact. The average period $\mathrm{T}_{\mathrm{osc}}$ of the contact bounce during the first 7 bounces is $3.8 \mu \mathrm{s}$. Apparently the kinetic energy of the switch at touchdown is not immediately dissipated, but excites eigenmodes of the membrane which persist for a substantial time. Eventually the kinetic energy is converted to heat and sound waves in the substrate.

To simulate the oscillation period after opening, we have calculated the eigenmodes of the beam suspended by springs in ANSYS. The lowest eigenmode is shown in figure 10a) with a corresponding eigenperiod of $19.6 \mu \mathrm{s}$ in quite good agreement with the measured period of $20.7 \mu$ s after release. To evaluate the period of the contact bounce effect we note that the observed bouncing motion is actually a capacitance change due to bending of the actuation plate. Because the spring force after collapse is much smaller than the electrostatic force, the springs can be neglected and the resonance is well approximated by just considering the eigenmodes of the actuation plate in free space. Indeed simulation shows that the actuation plate has an eigenmode with a period of $\mathrm{T}_{\text {eig }}=4.0 \mu \mathrm{s}$, close to the observed contact bounce period. The vertical displacement of this eigenmode is shown in figure $10 \mathrm{~b}$ ). The vertical displacement of the center of the 


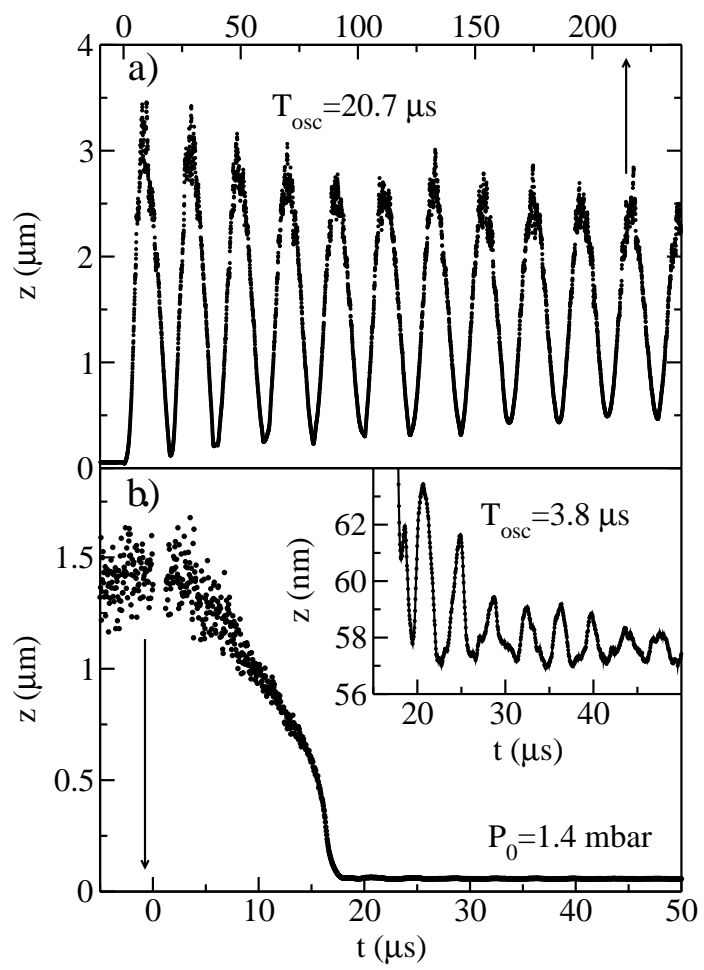

Figure 9. a) Opening motion of the switch at 1.4 mbar. b) Closing motion of the switch at 1.4 mbar, the inset shows a magnification of the motion after the switch has collapsed, which shows a periodic bouncing effect.

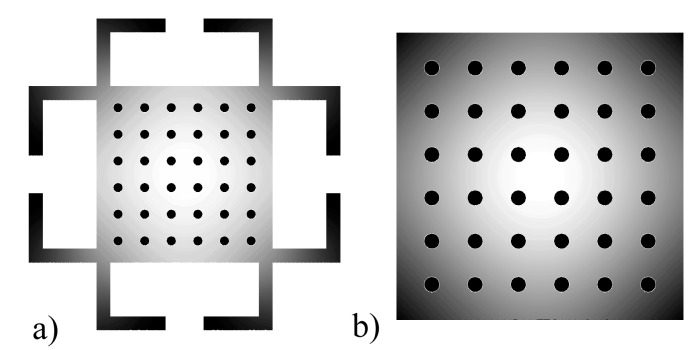

Figure 10. a) Lowest eigenmode of the anchored switch. The greyscale shows the vertical displacement, black is zero displacement, white is maximal displacement. $f_{\text {eig }}=51 \mathrm{kHz}, T_{\text {eig }}=19.6 \mu \mathrm{s}$. b) Eigenmode of the free centerplate which dominates the contact bouncing after collapse. White is $+38 \%$ and black $-62 \%$ of the total displacement. $f_{\text {eig }}=248 \mathrm{kHz}, T_{\text {eig }}=4.0 \mu$ s.

plate is $180^{\circ}$ out of phase with the corners and edges. The bouncing motion will be a superposition of eigenmodes, apparently the mode in figure 10b) is mainly responsible for the observed bouncing frequency.

For efficient switch operation, capacitance vibrations after opening as shown in figure 9a) are usually undesirable. On the other hand, overdamped behavior increases the switching time. To obtain smooth and quick settling to the equilibrium position, the damping should be adjusted to result in 'critical' damping. In a harmonic oscillator 
the damping force $F_{D}=b_{\text {harm }} v$ and the condition for critical damping is given by $b_{\text {crit,harm }}=2 \sqrt{\mathrm{km}}$. If the damping constant is above this value, no overshoot will occur. Because in a squeeze film damped system the damping constant depends on position, there is no simple expression for the critical damping coefficient. However as the damping force in a squeeze film damped system is at least $b v /\left((1+\kappa) g^{3}\right)$ an upper limit to the critical damping constant in a squeeze film damped system is given

by $b_{\text {crit,sqd }}=2(1+\kappa) g^{3} \sqrt{k m}$. For the switch under study $b_{\mathrm{FEM}}=1.04 \times b_{\text {crit,sqd }}$, it is therefore certainly in the overdamped regime and will not show an overshoot.

If a small overshoot can be allowed, faster opening can be achieved. This can be simulated by solving the equation of motion (17) numerically. Although this equation is difficult to solve analytically, a reasonable analytical estimate for the optimal damping constant can be found by minimizing the sum of the time $t_{\text {open }}$ it takes to reach the open position within a distance $z_{a}=\tilde{z}_{a} g$ using equation (18) and the time it takes for a harmonic oscillator damped with damping constant $b_{\text {harm }}=b /\left((1+\kappa) g^{3}\right)$ to reduce its amplitude below $z_{a}$. This results in an optimal damping constant $b_{\text {opt }}$ :

$$
b_{\mathrm{opt}}=\sqrt{\frac{2 m k g^{6}(1+\kappa)^{2} \ln \left(1 / \tilde{z}_{a}\right)}{\left.\tilde{t}_{\text {open }}\right|_{\tilde{z}_{0}} ^{1-\tilde{z}_{a}}}}
$$

Using this formalism, the damping constant to reach an amplitude $\tilde{z}_{a}=0.1$ in minimum time is for the switch under study given by $b_{\mathrm{opt}}=0.16 \times b_{\mathrm{FEM}}$.

\section{Conclusions}

A capacitive RF MEMS shunt switch was fabricated using the industrialized PASSI ${ }^{T M}$ process. The process yields very flat switches, which facilitates accurate modelling and reliable design. Using a high time-resolution setup, time dependent capacitance measurements and fast $\mathrm{C}-\mathrm{V}$ measurements were performed. The effect of dielectric charging on the $\mathrm{C}-\mathrm{V}$ curves was discussed. From the dynamic capacitance, the motion $z(t)$ of the switch could be calculated, using an analytic approximation to take fringe fields into account.

Squeeze film damping theory was discussed and an analytic equation to determine the damping coefficient of a perforated plate including edge effects was proposed, which compared well with finite element calculations. By differentiating the measured motion $z(t)$ the speed and acceleration of the switch were determined, which were used to extract the measured damping force at atmospheric pressure. Comparison with theory showed good agreement, both during closing and opening of the switch. It is concluded that the equation of motion of the electrostatic MEMS switch at atmospheric pressure is well described by equation (17). The presented framework of analytic equations including squeeze film damping can facilitate rapid modelling of gas damped MEMS switches.

Study of the motion of the switch at low pressure showed an underdamped harmonic resonance during its opening motion and periodic contact bouncing after closing. Both oscillation frequencies were in close agreement with simulated eigenfrequencies. 


\section{Acknowledgements}

We would like to thank J. Oostveen, J. van de Sande and H. Verberne for their skillful assistance. The work is supported by the European Union in the framework of the IST-2000-28231 project MEMS2TUNE.

\section{References}

[1] Rebeiz G M 2002 RF MEMS: Theory, Design and Technology (John Wiley \& Sons)

[2] van Beek J T M et al 2003 Proc. BCTM 2003, Toulouse pp 147-50

[3] Pulsford N 2002 RF Design magazine Nov. pp 40-8

[4] van Beek J T M et al 2003 MRS 2003, Boston

[5] Nieminen $\mathrm{H}$ et al 2001 European Patent Application 01660182.5-2203

[6] Peroulis D, Pacheco S P, Sarabandi K and Katehi L P B 2003 IEEE Transactions on Microwave Theory and Techniques $\mathbf{5 1}$ pp 259-70

[7] Jansman A B M, van Beek J T M, van Delden M H W M, Kemmeren A L A M and den Dekker A 2003, Proc. ESSDERC, Estoril, 16-18 Sept. 2003

[8] Chan E K, Garikipati K, Dutton R W 1999 J. MEMS 8 pp 208-17

[9] Reid J R 2002 Modeling and Simulation of Microsystems 2002 pp 250-3

[10] Lorrain P, Corson R and Lorrain F 1988 Electromagnetic Fields and Waves (W. H. Freeman and Company)

[11] Rottenberg X, Nauwelaers B, De Raedt W and Tilmans H A C 2004 Proc. 5th MEMSWAVE Workshop, Uppsala, 30 June-2 July 2004 pp B11-14

[12] Morse P M and Feshbach H 1953 Methods of Theoretical Physics (McGraw-Hill, New York)

[13] Griffin W S, Richardson H H and Yamanami S 1966 J. Basic Eng.-Trans. ASME 88 pp 451-6

[14] Sadd M H and Stiffler A K 1975 J. Eng. Ind.-Trans. ASME 97(4) pp 1366-70

[15] Blech J J 1983 J. Lubric. Tech.-Trans. ASME 105(4) pp 615-20

[16] Starr J B 1990 Solid-State Sensor and Actuator Workshop, 4th Technical Digest., IEEE , 4-7 June 1990 pp 44-7

[17] Andrews M, Harris I and Turner G 1993 Sensors and Actuators A 36 pp 79-87

[18] Veijola T, Kuisma H, Lahdenperä J and Ryhänen T 1995 Sensors and Actuators A 48 pp $239-48$

[19] Yang Y J, Gretillat M A and Senturia S D 1997 Proc. Int. Solid State Sensors and Actuators Conference (Transducers '97) Chicago 16-19 June 19972 pp 1093-6

[20] Kim E-S, Cho Y-H and Kim M-U 1999 Micro Electro Mechanical Systems, MEMS '99. Twelfth IEEE International Conference on , 17-21 Jan. 1999 pp 296-301

[21] Bao M, Yang H, Sun Y and French P J 2003 J. Micromech. Microeng. 13 pp 795-800

[22] Gupta R K and Senturia S D 1997 Micro Electro Mechanical Systems, 1997. MEMS '97, Proceedings, IEEE., Tenth Annual International Workshop on , 26-30 Jan. 1997 pp 290-4

[23] Oude Nijhuis M H H, Basten T G H, Wijnant Y H, Tijdeman H and Tilmans H A C 1999 Proc. Eurosensors XIII, The Hague, September 12-15, 1999 pp 1-4

[24] van Spengen W M, Puers R, Mertens R and De Wolf I 2002 Electron Devices Meeting, IEDM '02. Digest. International pp 901-4

[25] Veijola T, Tinttunen T, Nieminen H, Ermolov V and Ryhänen T 2002 Microwave Symposium Digest, IEEE MTT-S Int., 2-7 June 20022 pp 1213-6

[26] McCarthy B, Adams G G, McGruer N E and Potter D 2002 J. MEMS 11(3) pp 276-83

[27] Schrag G and Wachutka G 2002 Sensors and Actuators A 97-98 pp 193-200

[28] Mehner J E, Doetzel W, Schauwecker B and Ostergaard D 2003 Proc. Transducers '03, Solid-State Sensors, Actuators and Microsystems, 12th Int. Conf. on, June 9-12, 20032 pp 1840-3

[29] Škvor Z 1967 Acustica 19 pp 295-9 